\title{
Assessing Mammals' Abundance and Diversity Using Remotely Triggered Cameras in Similipal Tiger Reserve and Its Implications for Conservation
}

\author{
Hemanta Kumar Sahu* and Himanshu Sekhar Palei
}

\begin{abstract}
Knowledge of the presence and distribution of species is crucial for designing and evaluating conservation strategies within a region. Similipal is one of the first nine tiger reserves to be declared in 1973, one of the first eight biosphere reserve constituted in India in 1994. In view of its biodiversity and cultural richness it has been included in World Network Biosphere Reserve (WNBR) by UNESCO since May, 2009.In this study,diversity and abundance of medium to large sized mammals and forest disturbances were surveyed in Similipal Tiger Reserve. Protected populations of wild mammals sharing resources and habitat with livestock and human in this tropical forest of Similipal provide an opportunity to evaluate mammal abundances and their interaction with livestock and other anthropogenic factors.Diversity of medium to large sized mammals has assessed in Similipal Tiger Reserve by conducting 6,413 camera trap days of 187 trap stations, between November 2012 and July 2013. Out of 3,763 independent photographs, 24 mammal species were recorded from 1721 independent photographs.Eight globally threatened species recorded including the tiger and Asian elephant in our study area.Anthropogenic activities like illegal hunting, livestock grazing and free ranging domestic dog may be the detrimental factors for the mammalian species. These activities should be addressed through conservation and development perception, and will require an interdisciplinary approach cautiously incorporating social and ecological components.
\end{abstract}

Keywords--- Anthropogenic activity, Camera trap, Domestic dog, Mammal, Similipal Tiger Reserve

\section{INTRODUCTION}

Terrestrial mammals are a key component of tropical forest communities as indicators of ecosystem health and providers of important ecosystem services (Ahumada et al. 2011). However, monitoring such species in large remote forest areas is difficult due their elusive behavior and low abundances (Datta et al. 2008).Camera traps acknowledge important tools for monitoring nocturnal and cryptic species, and further development of their use has led to population estimation of natural marked animals by means of well consolidated capturerecaptures models (Karanth and Nichols 1998). However, the most reliable abundance estimation method capture-recapture

Post Graduate Department of Zoology, North Orissa University, Baripada, Odisha, India has difficult to achieve at larger spatial scales (Mackenzie et al. 2002), and it is only possible to identify individual natural marked animals. Therefore, for the majority of tropical animals, including ungulates, bears and other small mammals, it is not possible to identify individual with confidence. In this scenario, another approach has been proposed to apply abundance estimations with camera trapping data for a wider range species. In a simple and direct way, trapping rates (photographs/trapping effort) became widely used method in most studies (Trolle and Kéry 2005). Therefore, taking into account the caveats above, we estimated medium to large sized mammal abundances through RAI among fixed camera locations within our study area.

\section{STUDY AREA}

The study was conducted in Similipal Tiger Reserve (STR), Odisha, India covering an area of $2750 \mathrm{~km}^{2}$, with a core area of $1194.75 \mathrm{~km}^{2}$. The area lies within geographic coordinates of $20^{\circ} 17^{\prime}$ to $22^{\circ} 34^{\prime} \mathrm{N}$ latitude and $85^{\circ} 40^{\prime}$ to $87^{\circ} 10^{\prime} \mathrm{E}$ longitude. The area falls under the province of Chhotanagapur in Deccan Peninsula bio-geographic zone (Rodgers and Panwar 1988). Terrain of the area is undulating and hilly, whereas altitude ranges from 300 to 1,200 meters. Wikramanayake et al. (1998) classified the reserve as a Tropical moist deciduous forest (TMD).

\section{MATERIALS AND METHODS}

Between November 2012 and July 2013, we deployed camera traps covering the 16 forest ranges of the study area to estimate the status of animal.We divided the study area into 2 $\mathrm{km}^{2}$ grids and randomly chose grids for camera locations. Within the grid, cameras were predominantly set along park roads; at off-road locations, we installed along game trails and footpaths. Each station consisted of one camera trap of the Moultry D50. All camera traps were programmed to delay sequential photographs by 30 seconds and operate 24 hours per day, recorded time, date and temperature for each exposure. Camera traps were strapped to trees or stakes approximately $50 \mathrm{~cm}$ above ground and $1-2 \mathrm{~m}$ from the monitoring area. We aimed the censor parallel to the ground to monitor a colonial area approximately $1 \mathrm{~m}$ in diameter at $10 \mathrm{~m}$ distance. Cameras were checked at 10-14 day intervals for 
battery replacement and photo download. We aimed to leave camera traps in the forest for the 45 days, but due to work schedule conflicts, cameras were often picked up earlier or later.

The RAI was calculated forall camera traps mammal speciesand others based on formula following (O'Brien 2003):

$$
\mathrm{RAI}=\frac{\mathrm{A}}{\mathrm{N}} \times 100
$$

In which ' $A$ ' represents the total number of captures of a species by all cameras, and ' $N$ 'equals to the total camera traps days during the study period.

\section{RESULTS}

We conducted camera surveys at 187 locations (Fig. 1), resulting in 6,413 trap days (Mean: $34.48 \pm 10.55 \mathrm{SD}$, range: 9-51). Camera traps at an additional - locations did not yield data because they malfunctioned or were stolen, or damaged by poachers and elephants. Among the photographs, we identified 24 mammal species (domestic mammal excluded) and seven bird species. We classified 3,763 frames as independent photographs, of which $6.32 \% \quad(n=238)$ were carnivores, $39.41 \%(\mathrm{n}=1483)$ were non-carnivore mammals, $1.46 \%(n=55)$ were of birds, $25.4 \%(n=955)$ were villagers, $1.14 \%(n=43)$ were poachers, $16.2 \%(n=611)$ were staffs, and $8.03 \%(\mathrm{n}=302)$ were domestic animal. Among these domestic animal $44.37 \%(n=134)$ were domestic dogs. We could not determine species in $0.35 \%(n=13)$ of the photographs due to poor focus, lighting, or angle. The relative abundanceof animal is summarized in Table 1 . The detailed relative abundances of mammal, domestic animal and villagers of each forest range are given in Table 2. Among the mammal, two species were endangered, three were vulnerable and three were near threatened species as classified by the 2013 IUCN Red List of threatened species (IUCN 2013).

Based on camera traps, barking deer Muntiacusmuntjac was the most abundant species (RAI=6.5) followed by the wild boar Susscrofa (RAI=4.52) and hanuman langur Semnopithecus entellus (RAI=3.6)and the lowest abundance was tiger Pantheratigris, striped hyena Hyaenahyaena, Indian pangolin Manis crassicaudata and otter (RAI=0.3).The carnivore community was represented by 11 species in the tiger reserve, including four felids, two viverrids, two mustelids, one ursid, one hyaenid and one herpestid (Table 1). Among the globally threatened species, Asian elephant Elephas maximuswas the most abundant species (RAI=2.09) followed by the leopard Pantherapardus(RAI=1.68) and sambarRusa unicolor (RAI=1.39).

The relative abundance of anthropogenic activity photos were villagers $(\mathrm{RAI}=14.9)$, poachers $(\mathrm{RAI}=0.67)$, livestock $(\mathrm{RAI}=2.62)$ and dogs $(\mathrm{RAI}=2.09)$.

\section{DISCUSSION}

A comparison with a list of large and medium size potential species present in the study area (Annon. 2013) suggests that the completeness of our species recorded was $70.59 \%$ of the species potentially recorded during our survey. Some of the species were not recorded during our survey (Wild dog
Cuonalpinusand

Four-horned antelopeTetracerusquadricornis) may be locally rare as a result of hunting or as a result of widespread presence of human and domestic animals. However other specieslike Indian gray wolfCanis lupus pallipes, Golden jackal Canis aureus and Indian fox Vulpesbengalensis have been reported near human habitation of STR. The lack of records of these species may represent a relatively low local abundance.

The domestic dogs could also be a problem in the study area, where they accounted for $10.3 \%$ detection of anthropogenic photos in camera traps. The abundance and ranging behavior of domestic dogs are recognized as key factors determining their cumulative impacts on wild carnivore through exploitation, apparent and interface competition (Vanak and Gompper 2010). Dogs were accompanied by villagers and poachers in $48.5 \%$ and $8.21 \%$ respectively of all dog detections, and the same individual dogs were detected alone. It is possible that some of the dogs detected were feral, and their presence in the study area needs to be address.

\section{REFERENCES}

[1] Ahumada, Jorge A., Carlos E. F. Silva et al. 2001. Community structure and diversity of tropical forest mammals: data from a global camera trap network. Phil. Tans. R.. Soc. B, 366: 2703-2711. doi:10.1098/rstb.2011.0115

[2] Annon. 2012. Annual Report. Similipal Tiger Reserve, Odisha, India, pp $1-24$.

[3] Datta, A., M.O. Anand, R. Naniwadekar. 2008. Empty forests: Large carnivore and prey abundance in Namdapha National Park, north-east India. Biological Conservation, 141: 1429-1435.

[4] Karanth, K. U. \& Nichols, J. D. (1998). Estimation of tiger densities in India using photographic captures and recaptures. Ecology 79: 28522862.

[5] MacKenzie, D.I., Nichols, J.D., Lachman, G.B., Droege, S., Royle, J.A., Langtimm, C.A., 2002.Estimating site occupancy rates when detection probabilities are less than one. Ecology 83, 2248-2255.

[6] O'Brien, T.G., Kinnaird, M.F., and Wibisono, H.T. 2003. Crouching tigers, hidden prey: Sumatran tiger and prey populations in a tropical forest landscape. Animal Conservation 6:131-139.

[7] Trolle, M., Ke'ry, M., 2005. Camera-trap study of ocelot and other secretive mammals in the northern Pantanal. Mammalia 69, 405-412.

[8] Vanak, A.T., Gompper, M.E., 2010. Interference competition at the landscape level: the effect of free-ranging $\operatorname{dogs}$ on a native mesocarnivore. J. Appl. Ecol. 47, 1225-1232. 